\title{
A rare case of lymphomatoid granulomatosis occurring with ulcerative colitis
}

\begin{abstract}
Lymphomatoid granulomatosis ( $\mathrm{LyG})$ is a rare type of angiocentric and angiodestructive lymphoproliferative disorder. We report a rare presentation of lymphomatoid granulomatosis in a patient with ulcerative colitis who's been maintained on 6-Mercaptopurine for prolonged period of time. Although it is not clear whether inflammatory bowel disease (IBD) by itself is associated with such disorder or whether the use of immunosuppressive agents for the treatment of IBD induces EBV related lymphoproliferative disorder, it is important that clinicians are aware of the possible occurrence to ensure appropriate diagnostic evaluation and intervention.
\end{abstract}

Keywords: inflammatory bowel disease, lympho proliferative disorder, lymphomatoid granulomatosis

\author{
Volume 7 Issue 6 - 2017 \\ Samah Nassereddine, 1,2 Hind Rafei,' Imad \\ Tabbara, ${ }^{1,2}$ Timoty Dorouthy, ${ }^{1,3}$ Marie L \\ Borum ${ }^{1,3}$ \\ 'Department of Internal Medicine, George Washington \\ University School of Medicine and Health Sciences, USA \\ ${ }^{2}$ Division of Hematology/Oncology and GW Cancer Center, \\ George Washington University Medical Faculty Associates, USA \\ ${ }^{3}$ Division of Gastroenterology, George Washington University \\ Medical Faculty Associates, USA
}

\begin{abstract}
Correspondence: Marie L Borum, MD. Professor of Medicine, Director, Division of Gastroenterology, George Washington University Medical Faculty Associates, 2150 Pennsylvania Avenue, NW,Washington, DC 20037, USA, Tel | 202 74| 2222, Fax | 202 74I 2487,Emailsamah_nd@hotmail.com
\end{abstract}

Received: April 17, 2017 | Published: April 27, 2017

\section{Introduction}

Lymphomatoid granulomatosis (LyG) is a rare type of angiocentric and angiodestructive lymphoproliferative disorder, predominantly involving the lungs and other extranodal sites, such as the central nervous system (CNS). It is characterized by an abnormal proliferation and accumulation of lymphocytes on a background of polymorphonuclear cells in organ tissues causing damage to the blood vessels within the tissue. ${ }^{1}$ Lymphomatoid granulomatosis is considered to be a B cell process related to Epstein-Barr virus (EBV) infection and to date it is categorized under EBV associated lymphoproliferative disorders. ${ }^{1}$ The clinical presentation varies and usually mimics a vasculitis with systemic organ involvement, lungs being the primary involved organ. It has been rarely described in the literature orbital involvement as a primary involvement. There is good evidence that patients with immune dysregulation and patients on immunosuppressive therapy are at an increased risk of developing LyG. Only four reported cases of lymphomatoid granulomatosis were described in patients with crohn's disease and we are not aware of any reported LyG cases in patients with ulcerative colitis.

Although treatment for lymphomatoid granulomatosis remains challenging but it varies according to the grade of presentation from observation and holding the offending agent to rituximab based chemotherapy and interferon.

We report a rare presentation of LyG disease with primary ocular involvement in a patient with ulcerative colitis (UC) who's maintained on 6-Mercaptopurine. Our patients failed a rituximab based chemotherapy treatment.

\section{Case presentation}

A 36 year old manifestation with longstanding ulcerative colitis, well controlled with 6-MP, presented with left eye pain associated with increased tearing and crusting. Ophthalmologic evaluation revealed no evidence of uveitis or episleritis. He was treated with antibiotics and acyclovir for dacrocystitis following evidence of methicillin resistant staphylococcus aureus (MRSA) and herpes simplex virus (HSV) on a lacrimal duct swab. While on therapy, he developed bilateral blurred vision and diplopia for which he was referred to the university hospital for further evaluation. Upon transfer he noted a 6 pound unintentional weight loss during the past month. His examination was remarkable for right-sided lateral gaze palsy and a large left-sided cervical lymphadenopathy. Laboratory studies were only remarkable for a white count of 2000 and a C-reactive protein of 45. Extensive infectious disease workup included negative lyme serology, cryptococcal antigen; HSV, acid fast stain and rapid plasma regain (RPR). MRA / MRV of the brain revealed multifocal supratentorial and infratentorial hyperintense lesions with enhancement of the 5th and 6th cranial nerves. Cerebral spinal fluid (CSF) analysis showed a white count of 16 (100\% lymphocytic), glucose 35 , with a total protein of 178 and negative gram stain and culture. Flow cytometry on the CSF revealed a dominant population of small to large size lymphocytes, including a population of B lymphocytes representing $40-45 \%$ of all flow events and showing an abnormal immunophenotype with expression of CD43,CD 18 and lack of expression of CD20, and an apparent lambda light chain restriction with dim expression of surface immunoglobulin.

The cervical lymph node biopsy revealed CD4+ atypical lymphocytic infiltrates with extensive necrosis. The presence of EBV-encoded small RNA (EBER) by in situ hybridization (ISH) and the vascular invasion seen on pathology made the diagnosis of lymphomatoid granulomatosis. CT of the chest, abdomen and pelvis showed multiple cavitary nodular lesions with no lymphadenopathies or splenomegaly.

The patient was started on systemic corticosteroids in addition to Chemotherapy with EPOCH regimen (Etoposide, Prednisone, vincristine, cyclophosphamide, doxorubicin). Unfortunately, his disease course was complicated and he failed to respond to treatment. At that point, palliative were involved with transition to hospice. 


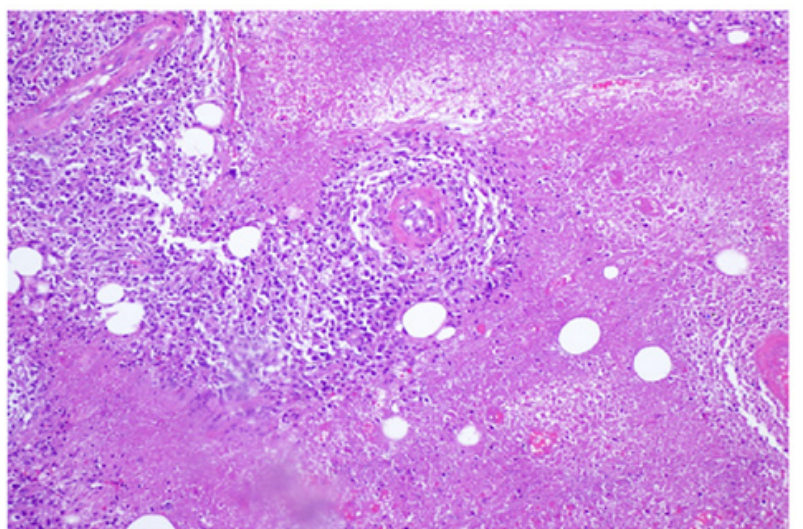

Figure I H\&E stain of a lymph node section demonstrating angiocentric infiltration of large atypical lymphocytes with prominent nuclei and extensive necrosis.

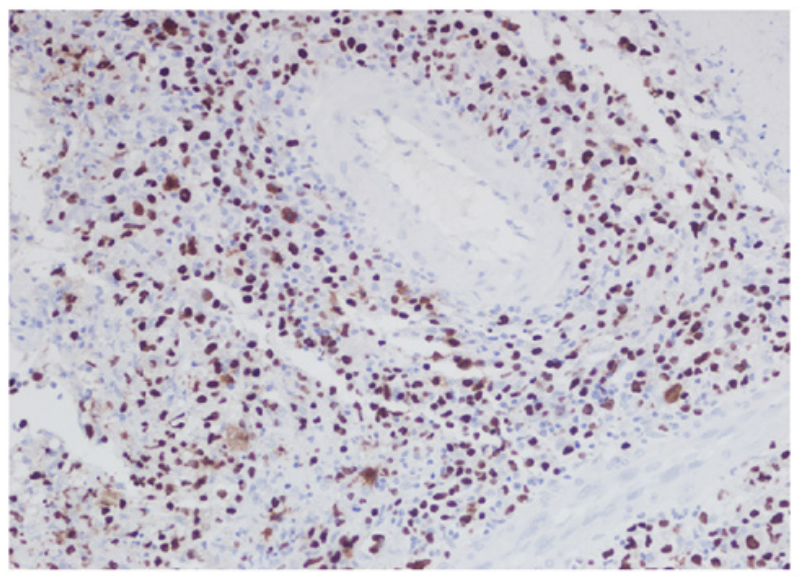

Figure 2 Immunohistochemical stain of the same lymph node showing numerous Ebstein Barr Virus Encoded RNA (EBER) positive cells.

\section{Discussion}

LyG was first described in 1972 by Liebow et al. ${ }^{1}$ based on the first 40 cases as an angiocentric and angiodestructive lymphoreticular proliferative and granulomatous disease involving predominantly the lungs. EBV was identified as the main mechanism in the pathogenesis of LyG. The causality was first described in 1990 when EBV DNA was found in tissue samples of 21 out of 29 patients with LYG. ${ }^{1}$

LyG has rarely been associated with inflammatory bowel disease. A review of the literature showed four reported cases. The previously reported individuals with IBD were noted to be in patients with Crohn's disease, all of whom were maintained on 6-Mercaptopurine and one was maintained on Azathioprine. ${ }^{2-5}$ Abdominal pain associated with B symptoms and respiratory symptoms was the common presentation. Diffuse systemic involvement including neurological lesions on imaging was also noted. The characteristic of these patients are summarized in table 1 with all the distinctive features and treatment offered.

The average age for the onset of LyG in the general population was noted to be 50 year old however the age of onset varies as we note two of the IBD patients were in the first two decades of life.

Our patient is the first patient with ulcerative colitis to be diagnosed with lymphomatoid granulomatosis. His initial presentation was ocular manifestation with dacryocystitis, ptosis and six nerve palsy. Orbital involvement has been described in 22 reported cases however only six patients had ocular manifestations as the initial presentation, four patients were noted to have ocular manifestation secondary to central nervous system involvement rather than direct orbital infiltration. ${ }^{6}$

LyG is considered to be an EBV driven process. Disease hypothesis suggests LyG arise in the setting of a dysregulation of EBV surveillance (deficit of EBV-specific CD8 T-cells). Progressive oncogenic events transform lower grade to higher grade disease: grades 1 and 2 are polyclonal or oligoclonal and immune dependent; grade 3 disease is monoclonal and immune independent. ${ }^{7}$

Although there is a concern for malignant lymphoma in patients with inflammatory bowel disease may be independent of the use of immunosuppressive agents, the use of such agents for the treatment of IBD increases the risk of developing lymphoma. The pathogenesis is still unclear and in one series, seven out of 18 patients with Azathioprine/6-Mercaptopurine-associated lymphomas were positive for the Epstein-Barr virus (EBV). Our patient most likely was at increased risk for LyG because of the immunosuppression from 6-MP triggering EBV infection.It's unclear whether inflammatory bowel disease independent of the use of immunosupressive agents, has an immunobiologic impact on the pathogenesis of lymphomatous granulomatosis.

Table I Comparative tables of all prior reported cases of LyG in patients with inflammatory bowel disease. 6FMP: 6FMercaptopurine; MTX: Methotrexate

\begin{tabular}{|c|c|c|c|c|}
\hline Cases & Case I & Case 2 & Case 3 & Case4 \\
\hline Disease & Crohn's disease & Crohn's disease & Crohn's disease & Crohn's disease \\
\hline $\begin{array}{l}\text { Age Presentation and } \\
\text { Gender }\end{array}$ & 7I Year old female & 26 year old female & 42 year old female & 17 year old female \\
\hline $\begin{array}{l}\text { Type of Presentation and } \\
\text { Diagnosis }\end{array}$ & Left sided Weakness & $\begin{array}{l}\text { Gastrointestinal, Lung } \\
\text { Involvement and Pancytopenia }\end{array}$ & Pulmonary involvement & $\begin{array}{l}\text { Cervical lymph node, Diarrhea and } \\
\text { Pulmonary involvement }\end{array}$ \\
\hline Home medications & $\begin{array}{l}\text { 6FMP and monthly } \\
\text { infliximab }\end{array}$ & Azathioprine for months & 6FMP for years & 6FMP for few months \\
\hline Treatment offered & $\begin{array}{l}\text { High dose } \\
\text { MTX }\end{array}$ & $\begin{array}{l}\text { Subcutaneous interferon Alpha } \\
\text { three times weekly and monthly } \\
\text { Rituximab }\end{array}$ & $\begin{array}{l}\text { Subcutaneous interferon } \\
\text { followed by R-EPOCH } \\
\text { followed by high dose } \\
\text { MTX }\end{array}$ & $\begin{array}{l}\text { Intermediate dose MTX and high dose } \\
\text { Cytosine } \\
\text { Arabinoside with a cycle of the protocol } \\
\text { Doxurubicin, Prednisone, Vincristine and } \\
6 \text { FMP }\end{array}$ \\
\hline Outcome & $\begin{array}{l}\text { Death after } 8 \\
\text { days }\end{array}$ & Alive & Death & Alive \\
\hline
\end{tabular}


Beaugerie et al. ${ }^{8}$ in their review showed an almost five-fold increased risk of lymphoproliferative disorders in crohn's disease patients receiving thiopurine therapy. ${ }^{8}$

While LyG is an unusual complication in IBD, it is important that clinicians are aware of the possible occurrence to ensure appropriate diagnostic evaluation and intervention.

Treatment for this condition remains controversial and outcome depends on the severity of presentation and extent of organ involved. Grading of these lesions is important because it dictates the treatment choice.

To date, there is no standardized treatment for patients with LyG, however in most cases, in individuals with low grade disease, discontinuation of thiopurine can result in remission, the use of a combination of corticosteroids and interferon-alpha can also achieve sustainable remissions. ${ }^{9}$ Aggressive malignancies similar to our patient may require multidrug chemotherapy. ${ }^{10}$

\section{Conclusion}

Here we present the first case in the literature of lymphomatoid granulomatosis in the setting of a patient with ulcerative colitis on 6-Mercaptopurine therapy. LyG is rare type of EBV-driven lymphoproliferative disorder. This case highlights the spectrum of clinical presentation of this condition. There is a need to increase awareness among clinicians to have higher clinical suspicion of this condition while evaluating IBD patients on immunomodulators. Future research is warranted in order to establish whether or not the use of immunomodulators increase the risk of LyG. Several ongoing studies evaluating different therapeutic approach to lymphomatoid granulomatosis with the hope to improve outcomes.

\section{Funding}

None.

\section{Acknowledgements}

None.

\section{Conflicts of interests}

The authors declare that there is no conflict of interest.

\section{References}

1. Katzenstein AL, Peiper SC. Detection of Epstein-Barr virus genomes in lymphomatoid granulomatosis: Analysis of 29 cases by the polymerase chain reaction technique. Modern Pathology. 1990;3(4):435-441.

2. Podolsky D, Gilberto Gonzalez R, Hasserjian. Case 8-2006: a 71-yearold woman with Crohn's disease and altered mental status. $N$ Engl J Med. 2006;354:1178-1184.

3. Connors W, Griffiths C, Patel J, et al. Lymphomatoid granulomatosis associated with azathioprine therapy in crohn disease. $B M C$ Gastroenterol. 2014;14:127.

4. Subramaniam K, Cherian M, Jain S, et al. Two rare cases of EpsteinBarr virus-associated lymphoproliferative disorders in inflammatory, bowel disease patients on thiopurines and other immunosuppressive medications. Int Med J. 2013;43(12):1339-1344.

5. Destombe S, Bouron-DalSoglio D, Rougemont AL, et al. Lymphomatoid granulomatosis: a unique complication of Crohn disease and its treatment in pediatrics. J Pediatr Gastroenterol Nutr. 2010;50(5):559-561.

6. Pradeep TG, Cannon P, Dodd T, et al. Lacrimal gland involvement in lymphomatoid granulomatosis and review of the literature. JOphthalmol .2010:358121

7. Dunleavy K, Roschewski M, Wilson WH. Lymphomatoid granulomatosis and other Epstein-Barr virus associated lymphoproliferative processes. Curr Hematol Malig Rep. 2012;7(3):208-215.

8. Lewis JD, Bilker WB, Brensinger C, et al. Inflammatory bowel disease is not associated with an increased risk of lymphoma. Gastroenterology. 2001;121:1080-1087.

9. Dunleavy K, Chattopadhyah P, Kawada J, et al. Immune characteristics associated with lymphomatoid granulomatosis and outcome following treatment with interferon-alpha. Blood. 2010;116: 963.

10. Gitelson E, Al-Saleem T, Smith MR. Review: lymphomatoid granulomatosis: challenges in diagnosis and treatment. Clin $A d v$ Hematol Oncol. 2009;7(1):68-70. 